International Journal of Pure and Applied Mathematics

Volume 104 No. 4 2015, 495-508

ISSN: 1311-8080 (printed version); ISSN: 1314-3395 (on-line version)

url: http://www.ijpam.eu

doi: http://dx.doi.org/10.12732/ijpam.v104i4.1

\title{
EFFICIENCY AND ACCURACY OF NUMERICAL SOLUTION OF LAGUERRE'S DIFFERENTIAL EQUATION USING HAAR WAVELET
}

\author{
Inderdeep Singh ${ }^{1}$, Sangeeta Arora ${ }^{2}$, Sheo Kumar ${ }^{3} \S$ \\ ${ }^{1,3}$ Department of Mathematics \\ Dr. B.R. Ambedkar National Institute of Technology \\ Jalandhar, Punjab, 144011, INDIA \\ ${ }^{2}$ PG Department of Computer Science and IT \\ HMV College \\ Jalandhar, 144008, INDIA
}

\begin{abstract}
From past literature, it is well known that Haar wavelet is a powerful mathematical tool for solving various type of differential equations and the solution obtained by Haar wavelet are more accurate than that obtained by other methods. Our aim in the present paper is to illustrate the slow computational convergence of Laguerre's differential equation using Haar wavelet, noting that Laguerre's differential equation has polynomial solutions.
\end{abstract}

AMS Subject Classification: 65L05, 34A45

Key Words: Laguerre's differential equation, Haar wavelet method, operational matrix

\section{Introduction}

Differential equations are used in many problems arising in mathematical, phys-

Received: April 10, 201

(c) 2015 Academic Publications, Ltd.

$\S$ Correspondence author url: www.acadpubl.eu 
ical and chemical sciences. During the last few decades considerable efforts have been made towards the development of computational procedure to differential equations encountered in various fields of science and engineering. Laguerre's differential equation is a special differential equation occuring in numerous engineering problems. The name of this differential equation is due to french mathematician Edmond Laguerre $(1834-86)$, who is known for his work in infinite series and geometry.

Consider the equation of the form

$$
x \frac{d^{2} y}{d x^{2}}+(1-x) \frac{d y}{d x}+n y=0,
$$

where $n$ is a constant, and is called the order of the differential equation. The most important property of this equation is that it has polynomial solution, naturally called Laguerre's polynomial. Laguerre's polynomials are the solution of equation (1). These polynomials $L_{n}(x)$ are given by:

$$
L_{n}=e^{x} \frac{d^{n}}{d x^{n}}\left(x^{n} e^{-x}\right) .
$$

The $L_{n}(x)$ can be obtained from the recurrence formula

$$
(n+1) L_{n+1}(x)=(2 n+1-x) L_{n}(x)-n L_{n-1}(x)
$$

In particular,

$$
\begin{gathered}
L_{0}(x)=1, \quad L_{1}(x)=1-x, \quad L_{2}(x)=1-2 x+\frac{1}{2} x^{2}, \\
L_{3}(x)=1-3 x+\frac{3}{2} x^{2}-\frac{1}{6} x^{3} .
\end{gathered}
$$

Their generating function is given as:

$$
\frac{e^{-x t / 1-t}}{1-t}=\sum_{n=0}^{\infty} \frac{L_{n}(x)}{n !} t^{n} .
$$

The orthogonal property of these polynomials is:

$$
\int_{0}^{\infty} e^{-x} L_{m}(x) L_{n}(x) d x= \begin{cases}0 & \text { for } m \neq n \\ (n !)^{2} & \text { for } m=n .\end{cases}
$$

In past literature $[9,10,11,12,13,14,15]$, it have been claimed that the solution of differential equations by Haar wavelet methods are more accurate. In the 
present paper, we see that the above claims by various authors in the past is not true for Laguerre's equation, even though it has polynomial solutions.

In Section 2, we briefly describe Haar wavelet method. In Section 3, we have given function approximation. Numerical methods for solving Laguerre's differential equation have been presented in Section 4 and in Section 5, numerical examples have been solved using the Haar wavelet method to illustrate the slow efficiency and accuracy of Laguerre's differential equation using Haar wavelet.

\section{Haar Wavelet Method}

In recent decades the field of Haar wavelets for solving differential equations has attracted interest of researchers in several areas of science and engineering. A survey on differential equation is presented in [15]. Wavelet analysis ia a new branch of mathematics and widely applied in differential and integral equations. The word wavelet is due to [7] in the early of 1980s. Several methods have been proposed to find the numerical solution of different linear and nonlinear differential equations. Wavelets have been applied extensively in mathematical problems related to scientific and engineering fields. There are many wavelet families such as Daubechies wavelet [8], Hermite-type trigonometric wavelet and many more. In 1910, Alfred Haar [2] introduced a function which presents a rectangular pulse pair. After that various generalizations were proposed. Among all these wavelet families, It is the simplest orthonormal wavelet with compact support. Haar wavelet is Daubechies wavelet of order one. Lepik $[10,12]$ presented the numerical solution of differential and integral equation with Haar wavelet method. The Haar functions are an orthogonal family of switched rectangular waveforms where amplitudes can differ from one function to another. They are defined in the interval $[0,1]$.

$$
h_{i}(x)= \begin{cases}1, & \alpha \leq x<\beta, \\ -1, & \beta \leq x<\gamma \\ 0, & \text { otherwise }\end{cases}
$$

where $\alpha=\frac{k}{m}, \beta=\frac{k+0.5}{m}$ and $\gamma=\frac{k+1}{m}$. Integer $m=2^{j},(j=0,1,2,3,4, \ldots \ldots . . J)$ indicates the level of the wavelet, and $k=0,1,2,3, \ldots ., m-1$ is the translation parameter. Maximal level of resolution is J. The index $i$ is calculated according the formula $i=m+k+1$. In the case of minimal values, $m=1, k=0$ we have $i=2$. The maximal value of $i$ is $i=2 M$, where $M=2^{J}$. It is assumed that 
the value $i=1$, corresponding to the scaling function in $[0,1]$.

$$
h_{1}(x)= \begin{cases}1, & 0 \leq x \leq 1 \\ 0, & \text { otherwise }\end{cases}
$$

Let us define the collocation points $x_{l}=\frac{(l-0.5)}{2 M}$, where $l=1,2,3, \ldots, 2 M$ and discretize the Haar function $h_{i}(x)$. Using the following notations of Haar functions:

$$
h_{1}(x)=[1,1,1,1], h_{2}(x)=[1,1,-1,-1], h_{3}=[1,-1,0,0], h_{4}=[0,0,1,-1] .
$$

we introduce the following notation:

$$
H_{4}(x)=\left[h_{1}(x), h_{2}(x), h_{3}(x), h_{4}(x)\right]^{T}=\left[\begin{array}{cccc}
1 & 1 & 1 & 1 \\
1 & 1 & -1 & -1 \\
1 & -1 & 0 & 0 \\
0 & 0 & 1 & -1
\end{array}\right]
$$

Here $H_{4}(x)$ is called Haar coefficient matrix. It is a square matrix of order 4 , and is defined as $H(i, l)=\left(h_{i}\left(x_{l}\right)\right)$, which has dimension $2 M \times 2 M$. Let us integrate equation (5), we get

$$
q_{i}(x)=\int_{0}^{x} h_{i}(t) d t
$$

In the collocation points, equation (8) gets the form $Q(i, l)=q_{i}\left(x_{l}\right)$, where $Q$ is a $2 M \times 2 M$ matrix. Chen and Hsiao [1] presented this matrix in the form $Q_{n}=P_{n} H_{n}$, where $P_{n} H_{n}$ is interpreted as the product of the matrices $P_{n}$ and $H_{n}$, called Haar integration and coefficient matrix, respectively. The operational matrix of integration $P$, which is a $2 M$ square matrix, is defined by the relations:

$$
\begin{aligned}
P_{i, 1}(x) & =\int_{0}^{x} h_{i}(t) d t . \\
P_{i, n+1}(x) & =\int_{0}^{x} P_{i, n}(t) d t,
\end{aligned}
$$

where $n=1,2,3,4 \ldots$ These integrals can also be evaluated using equation (5) as:

$$
P_{m, i}(x)= \begin{cases}0, & x<\alpha \\ \frac{1}{m !}\left\{(x-\alpha)^{m}\right\} & x \epsilon[\alpha, \beta) \\ \frac{1}{m !}\left\{(x-\alpha)^{m}-2(x-\beta)^{m}\right\} & x \epsilon[\beta, \gamma) \\ \frac{1}{m !}\left\{(x-\alpha)^{m}-2(x-\beta)^{m}+(x-\gamma)^{m}\right\} & x>\gamma\end{cases}
$$




\section{Function Approximation}

We know that all the Haar wavelets are orthogonal to each other:

$$
\int_{0}^{1} h_{i}(x) h_{l}(x) d x= \begin{cases}2^{-j} & i=l=2^{j}+k \\ 0 & i \neq l\end{cases}
$$

Therefore, they construct a very good transform basis. Any square integrable function $y(x)$ in the interval $[0,1]$ can be expanded by a Haar series of infinite terms:

$$
y(x)=\sum_{i=1}^{\infty} c_{i} h_{i}(x)
$$

where the Haar coefficients $c_{i}$ are determined as:

$$
\begin{gathered}
c_{0}=\int_{0}^{1} y(x) h_{0}(x) d x \\
c_{i}=2^{j} \int_{0}^{1} y(x) h_{i}(x) d x
\end{gathered}
$$

where $i=2^{j}+k, j \geq 0$ and $0 \leq k<2^{j}, x \epsilon[0,1]$ such that the following integral square error $\varepsilon$ is minimized:

$$
\varepsilon=\int_{0}^{1}\left[y(x)-\sum_{i=1}^{m} c_{i} h_{i}(x)\right]^{2} d x
$$

where $m=2^{j}$ and $j=0,1,2,3, \ldots$. Usually the series expansion of (13) contains infinite terms. If $y(x)$ is piecewise constant by itself or may be approximated as piecewise constant during each subinterval, then $y(x)$ will be terminated at finite $m$ terms. This means

$$
y(x) \cong \sum_{i=1}^{m} c_{i} h_{i}(x)=c_{m}{ }^{T} h_{m}(x)
$$

where the coefficients $c_{m}^{T}$ and the Haar function vectors $h_{m}(x)$ are defined as:

$$
c_{m}{ }^{T}=\left[c_{1}, c_{2}, c_{3}, \ldots, c_{m}\right] \text { and } h_{m}(x)=\left[h_{1}(x), h_{2}(x), h_{3}(x), \ldots, h_{m}(x)\right]^{T},
$$

where $T$ is the transpose. 


\section{Method for Solving Laguerre's Differential Equation}

Consider the Laguerre's differential equation (1) and assume that

$$
y^{\prime \prime}(x)=\sum_{i=1}^{2 M} a_{i} h_{i}(x)
$$

Integrating the above equation with respect to $x$, from 0 to $x$, we get,

$$
y^{\prime}(x)=y^{\prime}(0)+\sum_{i=1}^{2 M} a_{i} P_{1, i}(x)
$$

Again, Integrating the above equation with respect to $x$, from 0 to $x$, we get,

$$
y(x)=y(0)+x y^{\prime}(0)+\sum_{i=1}^{2 M} a_{i} P_{2, i}(x) .
$$

Substituting the values of $y(x), y^{\prime}(0)$ and $y^{\prime \prime}(0)$ in equation (1), we get

$$
\sum_{i=1}^{2 M} a_{i}\left[x h_{i}(x)+(1-x) P_{1, i}(x)+n P_{2, i}(x)\right]+(1-x) y^{\prime}(0)+n y(0)+n x y^{\prime}(0)=0 .
$$

From here, wavelet coefficients are calculated and we get the numerical solution of (1).

The accuracy of the results was estimated by the error function

$$
e_{J}=\max _{1 \leq i \leq 2 M}\left\{\left|y_{\text {approximate }}\left(x_{t}\right)-y_{\text {exact }}\left(x_{t}\right)\right|\right\}
$$

\section{Numerical Examples}

Here, we present numerical examples to observe the accuracy of Laguerre's differential equations by comparing the numerical solutions with exact solutions.

Example 1. Solve the differential equation

$$
x \frac{d^{2} y}{d x^{2}}+(1-x) \frac{d y}{d x}+2 y=0
$$




\begin{tabular}{|c|c|c|}
\hline $\mathrm{J}$ & $2 \mathrm{M}$ & Maximum absolute error \\
\hline 1 & 4 & 0 \\
\hline 2 & 8 & 0 \\
\hline 3 & 16 & $5.5 \mathrm{E}-017$ \\
\hline 4 & 32 & $5.5 \mathrm{E}-017$ \\
\hline 5 & 64 & $5.5 \mathrm{E}-017$ \\
\hline 6 & 128 & $1.1 \mathrm{E}-017$ \\
\hline 7 & 256 & $5.5 \mathrm{E}-017$ \\
\hline 8 & 512 & $5.5 \mathrm{E}-017$ \\
\hline
\end{tabular}

Table 1: Maximum absolute error $e_{j}$ for different values of $\mathrm{J}$

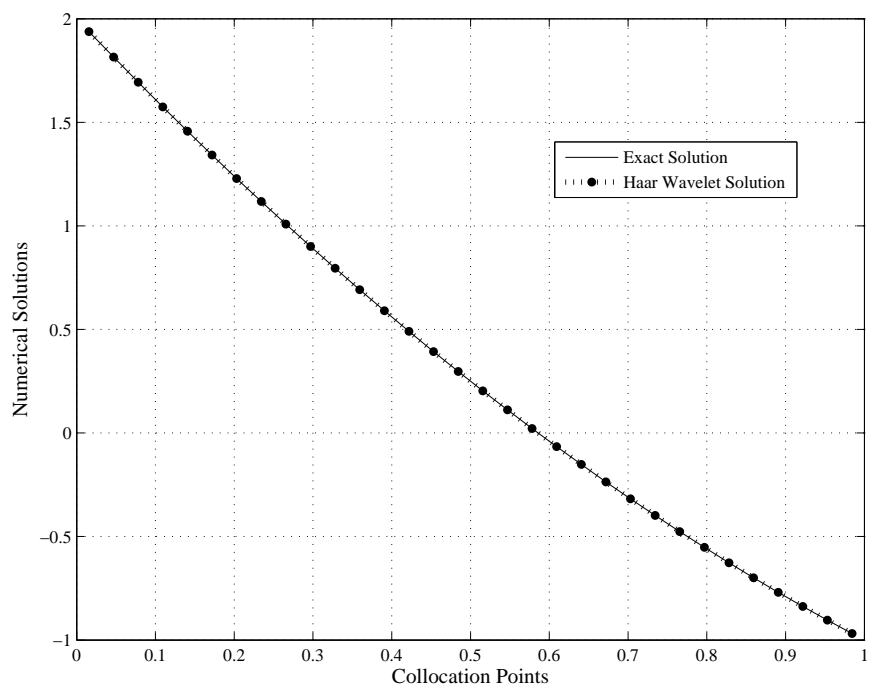

Figure 1: Comparison of numerical and exact solutions of Example 1 for $J=4$. 
with $y(0)=2$ and $y^{\prime}(0)=-4$. The exact solution of $(23)$ is $y(x)=1-2 x+\frac{1}{2} x^{2}$. Clearly, it is a Laguerre's differential equation of order 2. The maximum error functions of (23) at different values of $J$ are shown in the Table 1. Comparison of numerical and exact solutions of Example 1 for $J=4$ are shown in Figure 1.

Example 2. Solve the differential equation

$$
x \frac{d^{2} y}{d x^{2}}+(1-x) \frac{d y}{d x}+3 y=0
$$

with $y(0)=6$ and $y^{\prime}(0)=-18$. The exact solution of $(24)$ is $y(x)=1-3 x+\frac{3}{2} x^{2}-$ $\frac{1}{6} x^{3}$. Clearly, it is a Laguerre's differential equation of order 3 . The maximum error functions of (24) at different values of $J$ are shown in the Table 2. Comparison of numerical and exact solutions of Example 2 for $J=4$ are shown in Figure 2.

\begin{tabular}{|c|c|c|}
\hline $\mathrm{J}$ & $2 \mathrm{M}$ & Maximum absolute error \\
\hline 1 & 4 & $1.3 \mathrm{E}-003$ \\
\hline 2 & 8 & $0.3 \mathrm{E}-003$ \\
\hline 3 & 16 & $6.7 \mathrm{E}-005$ \\
\hline 4 & 32 & $1.5 \mathrm{E}-005$ \\
\hline 5 & 64 & $3.8 \mathrm{E}-005$ \\
\hline 6 & 128 & $9.3 \mathrm{E}-007$ \\
\hline 7 & 256 & $2.3 \mathrm{E}-007$ \\
\hline 8 & 512 & $5.7 \mathrm{E}-008$ \\
\hline
\end{tabular}

Table 2: Maximum absolute error $e_{j}$ for different values of $\mathrm{J}$

Example 3. Solve the differential equation

$$
x \frac{d^{2} y}{d x^{2}}+(1-x) \frac{d y}{d x}+4 y=0
$$

with $y(0)=24$ and $y^{\prime}(0)=-96$. The exact solution of $(25)$ is

$$
y(x)=1-4 x+3 x^{2}-\frac{2}{3} x^{3}+\frac{1}{24} x^{4} .
$$

Clearly, it is a Laguerre's differential equation of order 4. The maximum error functions of (25) at different values of $J$ are shown in the Table 3. Comparison of numerical and exact solutions of Example 3 for $J=4$ are shown in Figure 3.

Example 4. Solve the differential equation

$$
x \frac{d^{2} y}{d x^{2}}+(1-x) \frac{d y}{d x}+6 y=0
$$




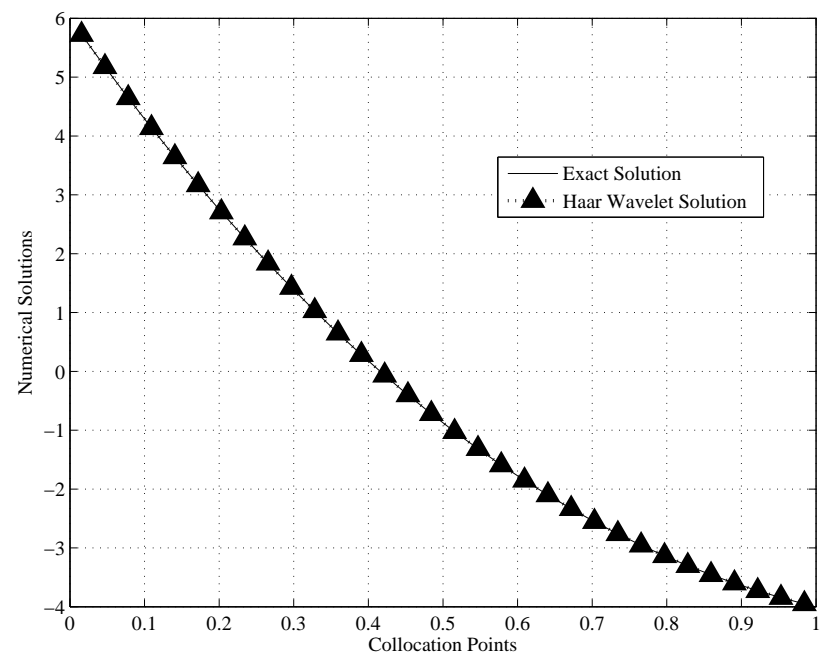

Figure 2: Comparison of numerical and exact solutions of Example 2 for $J=4$.

\begin{tabular}{|c|c|c|}
\hline $\mathrm{J}$ & $2 \mathrm{M}$ & Maximum absolute error \\
\hline 1 & 4 & $5.2 \mathrm{E}-003$ \\
\hline 2 & 8 & $1.0 \mathrm{E}-003$ \\
\hline 3 & 16 & $2.2 \mathrm{E}-004$ \\
\hline 4 & 32 & $5.1 \mathrm{E}-005$ \\
\hline 5 & 64 & $1.2 \mathrm{E}-005$ \\
\hline 6 & 128 & $3.0 \mathrm{E}-006$ \\
\hline 7 & 256 & $7.5 \mathrm{E}-007$ \\
\hline 8 & 512 & $1.8 \mathrm{E}-007$ \\
\hline
\end{tabular}

Table 3: Maximum absolute error $e_{j}$ for different values of $\mathrm{J}$ 


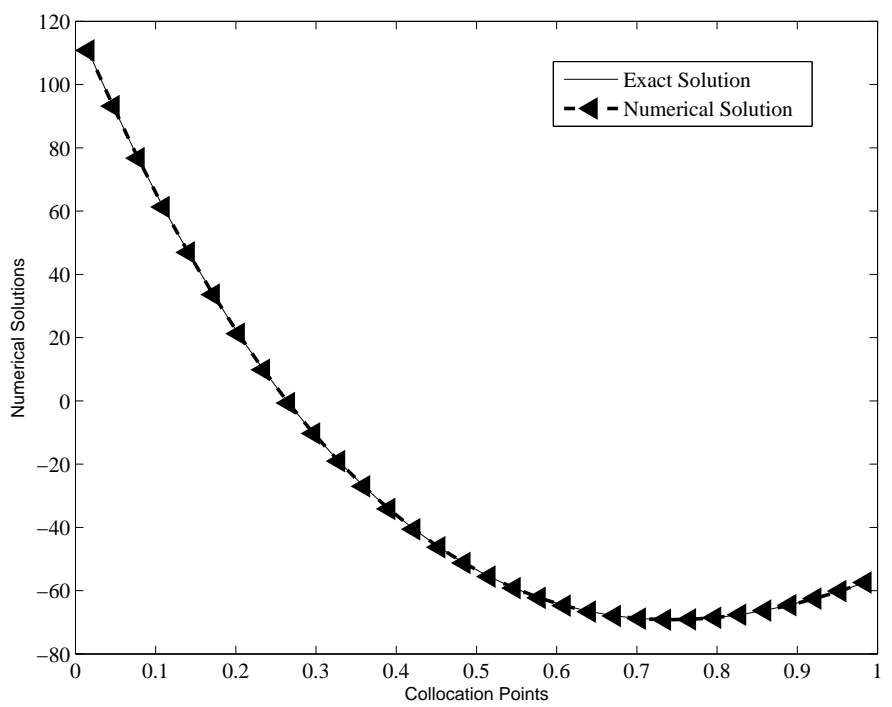

Figure 3: Comparison of numerical and exact solutions of Example 3 for $J=4$.

with $y(0)=720$ and $y^{\prime}(0)=-4320$.

The exact solution of (26) is

$$
y(x)=1-6 x+\frac{135}{18} x^{2}-\frac{10}{3} x^{3}+\frac{5}{9} x^{4}-36 x^{5}+\frac{1}{720} x^{6} .
$$

Clearly, it is a Laguerre's differential equation of order 6. The maximum error functions of (26) at different values of $J$ are shown in the Table 4. Comparison of numerical and exact solutions of Example 4 for $J=4$ are shown in Figure 4.

Example 5. Solve the differential equation

$$
x \frac{d^{2} y}{d x^{2}}+(1-x) \frac{d y}{d x}+7 y=0
$$

with $y(0)=5040$ and $y^{\prime}(0)=-35280$. The exact solution of $(28)$ is

$y(x)=1-7 x+\frac{52920}{5040} x^{2}-\frac{29400}{5040} x^{3}+\frac{7350}{5040} x^{4}-\frac{882}{5040} x^{5}+\frac{49}{5040} x^{6}-\frac{1}{5040} x^{7}$.

Clearly, it is a Laguerre's differential equation of order 7. The maximum error functions of (28) at different values of $J$ are shown in the Table 5. Comparison of numerical and exact solutions of Example 5 for $J=4$ are shown in Figure 5 . 


\begin{tabular}{|c|c|c|}
\hline $\mathrm{J}$ & $2 \mathrm{M}$ & Maximum absolute error \\
\hline 1 & 4 & $2.2 \mathrm{E}-002$ \\
\hline 2 & 8 & $3.8 \mathrm{E}-003$ \\
\hline 3 & 16 & $8.4 \mathrm{E}-004$ \\
\hline 4 & 32 & $1.9 \mathrm{E}-004$ \\
\hline 5 & 64 & $4.4 \mathrm{E}-005$ \\
\hline 6 & 128 & $1.0 \mathrm{E}-005$ \\
\hline 7 & 256 & $2.6 \mathrm{E}-006$ \\
\hline 8 & 512 & $6.6 \mathrm{E}-007$ \\
\hline
\end{tabular}

Table 4: Maximum absolute error $e_{j}$ for different values of $\mathrm{J}$

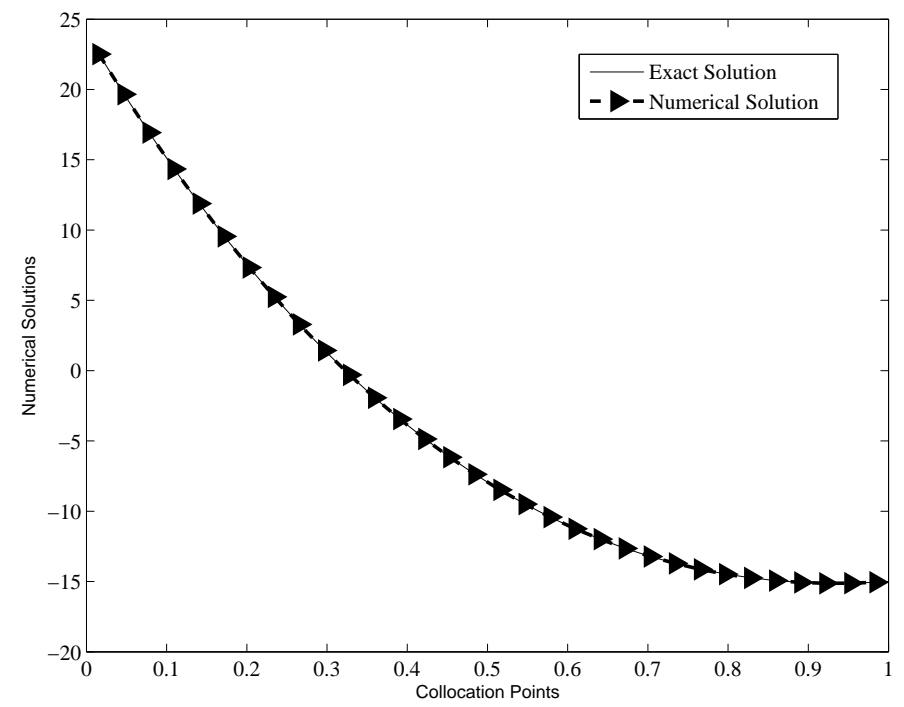

Figure 4: Comparison of numerical and exact solutions of Example 4 for $J=4$. 


\begin{tabular}{|c|c|c|}
\hline $\mathrm{J}$ & $2 \mathrm{M}$ & Maximum absolute error \\
\hline 1 & 4 & $3.5 \mathrm{E}-002$ \\
\hline 2 & 8 & $6.5 \mathrm{E}-003$ \\
\hline 3 & 16 & $1.3 \mathrm{E}-003$ \\
\hline 4 & 32 & $2.9 \mathrm{E}-004$ \\
\hline 5 & 64 & $6.8 \mathrm{E}-005$ \\
\hline 6 & 128 & $1.6 \mathrm{E}-005$ \\
\hline 7 & 256 & $4.0 \mathrm{E}-006$ \\
\hline 8 & 512 & $1.0 \mathrm{E}-006$ \\
\hline
\end{tabular}

Table 5: Maximum absolute error $e_{j}$ for different values of $\mathrm{J}$

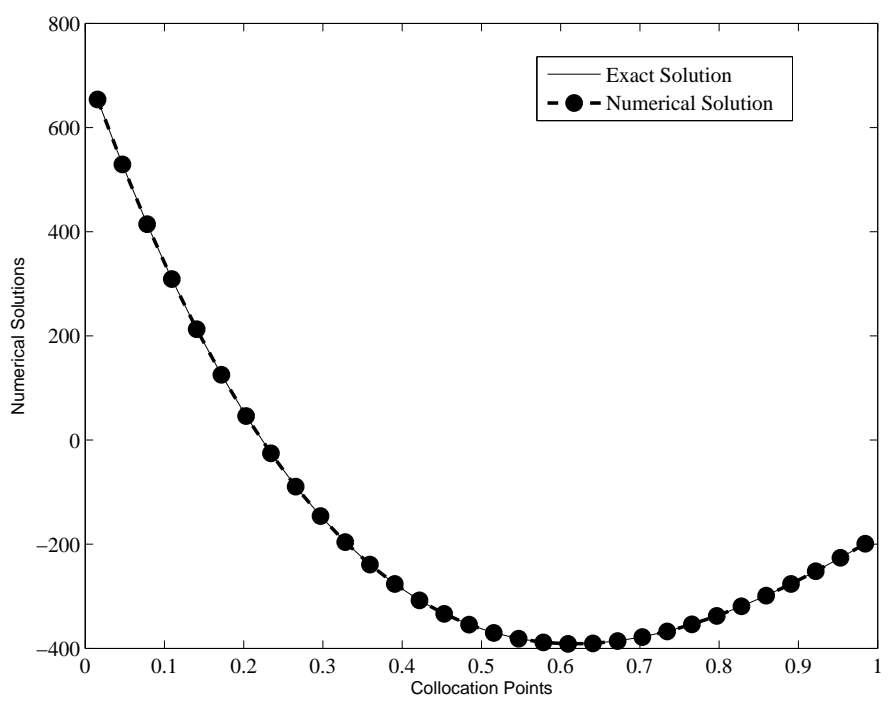

Figure 5: Comparison of numerical and exact solutions of Example 5 for $J=4$. 


\section{Conclusion}

We see that the analytical solution of $n$th order Laguerre's differential equation is $n$th degree polynomial. As the value of $n$ increases, the accuracy of the solution by the Haar wavelet method decreases rapidly. If this is happening for a polynomial solution of a differential equation, nothing can be said about the numerical solution of other differential equations having poor smooth/nonsmooth solutions. Thus, in view of the above it can not be accepted that Haar wavelet gives more accurate numerical solutions. We may see the work done in $[3,4,5,6]$ and the computational convergence/accuracy of numerical results obtained there. The computational convergence/accuracy of the results obtained in $[3,4,5,6]$ are far better than one obtained here. Thus it will be interesting to study for what class of differential equations, the Haar wavelet will give better results.

\section{References}

[1] C.F. Chen and C.H. Hsiao, Haar wavelet method for solving lumped and distributed-parameter systems, IEE Proc., Control Theory Appl., 144 (1997) 87-94.

[2] A. Haar, Zur theorie der orthogonalen Funktionsysteme, Math. Ann. 69 (1910) 331-371.

[3] M. M. Chawla and Sheo Kumar, Convergence of quadratures for Cauchy principal value integrals, Computing 23 (1979) 67-72.

[4] Sheo Kumar, A note on quadratures for Cauchy principal value integrals, Journal of the Institute of Mathematics and its Applications 26 (1980) 447-451.

[5] Sheo Kumar, On a method of Noble for second kind Volterra integral equations, BIT 19 (1979) 482-488.

[6] Sheo Kumar, On modified increment methods of Garey for nonlinear second kind Volterra integral equations, BIT 20 (1980) 346-350.

[7] A. Grossmann and J. Morlet, Decomposition of Hardy functions into square integrable wavelets of constant shape, SIAM, J. Math. Anal. 15 (1984) 723-736. 
[8] I. Daubechies, Ten Lectures on wavelets, CBMS-NCF, SIAM, Philadelphia, (1992).

[9] Ü. Lepik, Numerical solutionof differential equations using Haar wavelet, Math. Comp. in Simulation 68 (2003) 127-143.

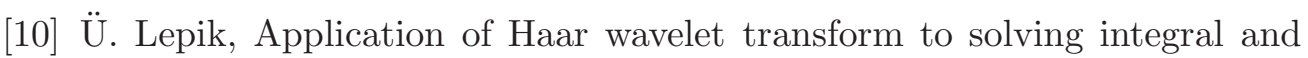
differential equations, Proc. Estonian Acad. Sci. Phys. Math., 56(1) (2007) 28-46.

[11] Ü. Lepik, Haar wavelet methods for solving higher order differential equations, International Journal of Mathematics and Computations 1 (2008) 84-94.

[12] Ü. Lepik, Solving differential and integral equations by Haar wavelet method, Revisted, International Journal of Mathematics and Computations 1 (2008) 43-52.

[13] G. Hariharan, K. Kannan and K.R. Sharma, Haar wavelet method for solving Fisher's equation, Applied Mathematics and Computation 211 (2009) 284-292.

[14] G. Hariharan and K. Kannan, Haar wavelet method for solving some nonlinear Parabolic equation, J. Math. Chem. 48 (2010) 1044-1061.

[15] G. Hariharan, An overview of Haar wavelet method for solving differential and integral equations, World Applied Sciences Journal, 23 (2013) 01-14. 